\title{
EFEITO AGUDO DE DIFERENTES PROTOCOLOS DE PRÉ-CONDICIONAMENTO ISQUÊMICO EM UMA PROVA DE 4KM DE CICLISMO CONTRARRELÓGIO
}

\author{
Gabriel A. Truffi ${ }^{\star}$, Renato Barroso
}

\section{Resumo}

O objetivo do estudo foi analisar os efeitos agudos de protocolos de pré-condicionamento isquêmico ( $\mathrm{PCl}$ ) com diferentes números de ciclos e intervalo antes da prática sobre o desempenho em uma prova de $4 \mathrm{~km}$ contrarrelógio de ciclismo. Para tanto, participaram do estudo 15 homens ciclistas treinados e, em um design crossover, foram combinados 1, 2 ou 4 ciclos (CIC) de PCl com intervalo (INT) antes da prática de 15 ou 30 minutos e também uma condição controle (CTRL), sem qualquer intervenção de PCl. O tempo para conclusão dos $4 \mathrm{~km}$, em cada condição, foi de: $1 \mathrm{CIC}+15 \mathrm{INT}: 383,2 \mathrm{~s} \pm 31 ; 1 \mathrm{CIC}+30 \mathrm{INT}: 383,7 \mathrm{~s} \pm 32,6 ; 2 \mathrm{CIC}+15 \mathrm{INT}: 386,6 \mathrm{~s} \pm 35,1 ; 2 \mathrm{CIC}+30 \mathrm{INT}: 383,1 \mathrm{~s} \pm$ 30,$1 ; 4 \mathrm{CIC}+15 \mathrm{INT}: 382,4 \mathrm{~s} \pm 33,6 ; 4 \mathrm{CIC}+30 \mathrm{INT}: 382,4 \mathrm{~s} \pm 33 ; \mathrm{CTRL}: 384,7 \mathrm{~s} \pm 34$. A análise estatística feita a partir de uma Anova one-way nos mostrou que não houve diferença significativa entre as condições de $\mathrm{PCl}$ em relação à CTRL, considerando $\mathrm{p}<0,05$. Portanto, nenhum dos protocolos de PCl foi capaz de melhorar a performance dos atletas nos $4 \mathrm{~km}$ de ciclismo contrarrelógio.

\section{Palavras-chave: desempenho, ciclismo, pré-condicionamento isquêmico.}

\section{Introdução}

O pré-condicionamento isquêmico $(\mathrm{PCl})$ se trata de uma prática na qual é realizado um período de isquemia, seguido de uma reperfusão do fluxo sanguíneo. Atualmente, o $\mathrm{PCl}$ vem sendo muito investigado em estudos no âmbito esportivo, com diversos estudos mostrando melhoras na performance de atividades com predominância de metabolismo aeróbio. Porém, apesar de mostrar benefícios à performance, o tradicional protocolo de $\mathrm{PCl}$ com 4 ciclos e 30 minutos de intervalo antes da prática demanda um longo tempo. Assim, realizamos um total de 7 sessões experimentais, combinando 1,2 ou 4 ciclos de $\mathrm{PCl}$ com 15 ou 30 minutos de intervalo prévio ao exercício, e também uma condição CTRL, sem qualquer intervenção de $\mathrm{PCl}$. Desse modo, o objetivo desse estudo foi testar diferentes protocolos de $\mathrm{PCl}$ para identificar se mesmo os mais curtos seriam benéficos à performance de ciclistas em uma prova de $4 \mathrm{~km}$ de ciclismo contrarrelógio.

\section{Resultados e Discussão}

Participaram do estudo 15 homens ciclistas treinados $(30,7 \pm 6$ anos de idade, $179 \pm 6,6 \mathrm{~cm}$ de altura e 74,6 \pm $10,1 \mathrm{~kg}$ de massa corporal).

Os tempos de conclusão da prova de $4 \mathrm{~km}$ se encontram na tabela abaixo.

Tabela 1. Tempo para conclusão dos $4 \mathrm{~km}$ contrarrelógio.

\begin{tabular}{|c|c|}
\hline Condição & Tempo \\
\hline $1 \mathrm{CIC}+15 \mathrm{INT}$ & $383,2 \mathrm{~s} \pm 31$ \\
\hline $1 \mathrm{CIC}+30 \mathrm{INT}$ & $383,7 \mathrm{~s} \pm 32,6$ \\
\hline $2 \mathrm{CIC}+15 \mathrm{INT}$ & $386,6 \mathrm{~s} \pm 35,1$ \\
\hline $2 \mathrm{CIC}+30 \mathrm{INT}$ & $383,1 \mathrm{~s} \pm 30,1$ \\
\hline $4 \mathrm{CIC}+15 \mathrm{INT}$ & $382,4 \mathrm{~s} \pm 33,6$ \\
\hline $4 \mathrm{CIC}+30 \mathrm{INT}$ & $382,4 \mathrm{~s} \pm 33$ \\
\hline $\mathrm{CTRL}$ & $384,7 \mathrm{~s} \pm 34$ \\
\hline
\end{tabular}

Na comparação entre as condições de PCl e CRTL, foi feita uma Anova one-way para medidas repetidas. O nível de significância adotado foi de $p<0,05$. Assim sendo, não houve diferença estatística significativa em nenhuma das comparações.

Relacionando nossos resultados à alguns estudos de $\mathrm{PCl}$ e exercícios físicos, tivemos algumas ideias do motivo pelo qual não encontramos melhoras na performance.
A primeira possibilidade é de que a pressão do manguito deve ser aplicada de maneira simultânea em ambos os membros inferiores do indivíduo. No trabalho de Kilding ${ }^{1}$, com o $\mathrm{PCl}$ realizado com 4 ciclos de maneira simultânea e também em uma prova de $4 \mathrm{~km}$ contrarrelógio de ciclismo, foram encontradas melhoras no tempo de conclusão da prova. Já o trabalho de Clevidence ${ }^{2}$, conduzido com 3 ciclos de PCl de maneira alternada nos membros inferiores, não encontrou benefícios na performance aeróbia ou anaeróbia em um teste de esforço submáximo no ciclismo.

Outra incongruência com o estudo de Kilding ${ }^{1}$ é que o mesmo foi feito com intervalo de apenas 5 minutos entre o final dos ciclos de $\mathrm{PCl}$ para 0 início da atividade. Entretanto, de acordo com Das e Das ${ }^{3}$, há ao menos duas janelas para os efeitos positivos do $\mathrm{PCl}$ na medicina. A primeira se inicia com alguns minutos do término dos ciclos de $\mathrm{PCl}$ e pode durar até 2 horas. Assim, em todas as nossas condições, trabalhamos dentro dessa janela. Porém, ainda não há evidências suficientes para dizer se de fato essas janelas são as mesmas para uma melhora em relação à performance em exercícios físicos.

\section{Conclusões}

Nossos resultados indicam que nenhum dos protocolos de $\mathrm{PCl}$ foi capaz de induzir melhoras significativas na performance de ciclistas homens treinados em uma prova de $4 \mathrm{~km}$ contrarrelógio.

\section{Agradecimentos}

Agradecemos ao Laboratório de Estudos Eletromiográficos (LEE) e ao LABFEF pelo espaço e equipamentos disponibilizados e ao CNPq/PIBIC pelo financiamento do projeto.

\footnotetext{
${ }^{1}$ KILDING, A. E.; SEQUEIRA, G. M.; WOOD, M. R. Effects of ischemic preconditioning on economy, VO 2 kinetics and cycling performance in endurance athletes. European journal of applied physiology, v. 118, n. 12, p. 2541-2549, 2018.

2 CLEVIDENCE, Michael W.; MOWERY, Robert E.; KUSHNICK, Michael $\mathrm{R}$. The effects of ischemic preconditioning on aerobic and anaerobic variables associated with submaximal cycling performance. European journal of applied physiology, v. 112, n. 10, p. 3649-3654, 2012.

3 DAS, Manika; DAS, Dipak K. Molecular mechanism of preconditioning. IUBMB life, v. 60, n. 4, p. 199-203, 2008.
} 